\title{
Time Intervals of the Energy Emission in Quantum Systems Obtained from the Conservation Rule of the Electron Momentum
}

\author{
Stanisław Olszewski \\ Institute of Physical Chemistry, Polish Academy of Sciences, Warsaw, Poland \\ Email: olsz@ichf.edu.pl
}

How to cite this paper: Olszewski, S. (2021) Time Intervals of the Energy Emission in Quantum Systems Obtained from the Conservation Rule of the Electron Momentum. Journal of Modern Physics, 12, 661-670.

https://doi.org/10.4236/jmp.2021.125043

Received: March 12, 2021

Accepted: April 26, 2021

Published: April 29, 2021

Copyright () 2021 by author(s) and Scientific Research Publishing Inc. This work is licensed under the Creative Commons Attribution International License (CC BY 4.0).

http://creativecommons.org/licenses/by/4.0/

\begin{abstract}
The paper presents a non-probabilistic approach to the time interval associated with the energy emission produced by the electron transition in a quantum system. The calculations were performed for the hydrogen atom and the electron particle in a one-dimensional potential box. In both cases, the rule of conservation of the electron momentum has been applied. The results, limited to the time intervals of transitions between two neighbouring quantum energy levels, occur to be much similar to those obtained earlier with the aid of the Joule-Lenz energy emission theory.
\end{abstract}

\section{Keywords}

Non-Probabilistic Approach to the Electron Transition Time, Conservation Rule of the Electron Momentum, Joule-Lenz Energy Emission Theory

\section{Introduction}

In principle, we assume that some interval of time should accompany any quantum process in which a change of a quantum physical system does occur. In the previous approach to such processes, a probabilistic analysis accompanied any electron transition phenomenon (see e.g. [1] [2] [3] [4]), leaving unknown the corresponding interval, or intervals, of time. In general, such situation did not change in the modern quantum theory [5] [6].

A different, viz. non-probabilistic situation, took place when the classical Joule-Lenz theorem for the energy emission (see e.g. [7]) has been adapted in calculating the transition time of an electron between two quantum energy levels [8]-[14]. In this case, a very simple rule coupling the distance between two quantum energy levels with the size of the time interval for the electron transi- 
tion could be found. Nevertheless, the limitations of that rule became rather evident. In consequence, an alternative approach to the time interval of the electron transition seemed to be of use. Such approach is outlined in the next Sections of the present paper.

\section{Electron Momentum, Its Change and a Use of the Postulate Concerning Conservation of the Momentum. The Case of the Hydrogen Atom Taken as an Example}

In principle any change of the electron energy, say obtained in effect of the electron transition between two quantum levels, can be associated with a corresponding change of the electron momentum. By taking into account the bound electron states of the hydrogen atom, the state $n$ of the energy is given by [15] [16]

$$
E_{n}=-E_{\mathrm{kin}}^{(n)}=-\frac{m}{2} v_{n}^{2},
$$

where the first equation in (1) is due to the virial theorem

$$
2 E_{\text {kin }}^{(n)}+E_{\text {pot }}^{(n)}=0
$$

in result of which the total electron energy of the atom equal to a sum of the kinetic and potential parts becomes

$$
E_{\mathrm{tot}}^{(n)}=E_{\mathrm{kin}}^{(n)}+E_{\mathrm{pot}}^{(n)}=E_{n} .
$$

The electron velocity entering (1) is [17]

$$
v_{n}=\frac{e^{2}}{n \hbar}
$$

and the electron momentum in state $n$ becomes

$$
p^{n}=m v_{n} .
$$

Another approach to $p^{n}$ can be obtained from the quanta of the electron angular momentum

$$
L_{n}=m v_{n} r_{n}=\frac{m e^{2}}{n \hbar} \frac{n^{2} \hbar^{2}}{m e^{2}}=n \hbar
$$

which holds because

$$
r_{n}=\frac{n^{2} \hbar^{2}}{m e^{2}}
$$

is the radius of the electron circular orbit in the hydrogen atom [17].

A final result for the quanta of energy in (1) is

$$
E_{n}=-\frac{m}{2} \frac{e^{4}}{n^{2} \hbar^{2}}
$$

and the energy change

$$
\Delta E=E_{n+1}-E_{n}
$$

due to the change of the quantum state is equal to 


$$
\Delta E=\frac{m}{2} \frac{e^{4}}{\hbar^{2}}\left[\frac{1}{n^{2}}-\frac{1}{(n+1)^{2}}\right]=\frac{m}{2} \frac{e^{4}}{\hbar^{2}} \frac{(n+1)^{2}-n^{2}}{n^{2}(n+1)^{2}}=\frac{m e^{4}}{\hbar^{2}} \frac{n+1 / 2}{n^{2}(n+1)^{2}} \cong \frac{m e^{4}}{\hbar^{2}} \frac{1}{n^{3}} .
$$

The last step in (10) is valid on condition $n$ is a large number.

Respectively to (9) we have the momentum change

$$
\Delta p^{n}=p^{n+1}-p^{n}=\frac{m e^{2}}{\hbar}\left(\frac{1}{n+1}-\frac{1}{n}\right)=-\frac{m e^{2}}{\hbar} \frac{1}{(n+1) n} \approx-\frac{m e^{2}}{\hbar n^{2}}
$$

which provides us evidently with a smaller electron momentum in state $n+1$ than in state $n$.

If the momentum in states $n+1$ and $n$ should be conserved, the negative difference in (11) has to be compensated by the momentum supplement resulting from the orbit change, viz.

$$
\Delta r_{n}=r_{n+1}-r_{n},
$$

in effect of which we obtain the momentum change

$$
m \frac{\Delta r_{n}}{\Delta t}=m \frac{r_{n+1}-r_{n}}{\Delta t}=\frac{m}{\Delta t}\left[\frac{(n+1)^{2} \hbar^{2}}{m e^{2}}-\frac{n^{2} \hbar^{2}}{m e^{2}}\right]=\frac{(2 n+1) \hbar^{2}}{\Delta t e^{2}} \approx \frac{2 n \hbar^{2}}{\Delta t e^{2}} .
$$

We postulate that the sum of (11) and (13) has to be zero, so

$$
\Delta p_{n}+m \frac{\Delta r_{n}}{\Delta t}=0
$$

or

$$
-\frac{m e^{2}}{\hbar n^{2}}+\frac{2 n \hbar^{2}}{\Delta t e^{2}}=0
$$

It should be noted that the momenta balance postulated in (15) concerns solely the momenta values and not directions of the vectors. The requirement in (15) gives

$$
\frac{m e^{2}}{\hbar n^{2}}=\frac{2 n \hbar^{2}}{\Delta t e^{2}}
$$

from which

$$
\Delta t=\frac{2 n^{3} \hbar^{3}}{m e^{4}}
$$

This $\Delta t$ is a time interval necessary to provide us with a conservation of momentum represented by the formula (14).

\section{Comparison with the Joule-Lenz Law [8]-[14]}

According to that law the time interval

$$
\Delta t^{(J L)}
$$

should approximately satisfy the formula

$$
\Delta E \Delta t^{(J L)}=\frac{m e^{4}}{\hbar n^{3}} \Delta t^{(J L)}=h,
$$


where the interval $\Delta E$ is taken from (10). In virtue of (19) we have

$$
\Delta t^{(J L)}=\frac{2 \pi \hbar^{3} n^{3}}{m e^{4}} .
$$

In result we find that the Joule-Lenz emission time (20) differs from the time interval obtained in (17) solely by the factor of $\pi$.

\section{Time Interval Connected with the Absorption of Energy Compared with the Time of the Emission Process}

Both the absorption and emission processes are of a semiclassical nature. Therefore if in case of absorption we have a change of quantum indices

$$
n \rightarrow n+1
$$

the result for the time interval $\Delta t$ becomes equal to that for the case of emission between the levels

$$
n+1 \rightarrow n
$$

A different situation can be obtained when the emission change of states

$$
n \rightarrow n-1
$$

is compared with the absorption change which is for example

$$
n \rightarrow n+1 \text {. }
$$

In the case of (23) we have the momentum balance given by the condition

$$
\begin{aligned}
p^{n-1}-p^{n}+m \frac{r_{n-1}-r_{n}}{\Delta t} & =\frac{m e^{2}}{\hbar}\left(\frac{1}{n-1}-\frac{1}{n}\right)+m \frac{(n-1)^{2}-n^{2}}{\Delta t} \frac{\hbar^{2}}{m e^{2}} \\
& =\frac{m e^{2}}{\hbar} \frac{1}{(n-1) n}+\frac{m}{\Delta t}(-2 n+1) \frac{\hbar^{2}}{m e^{2}}=0
\end{aligned}
$$

from which we obtain the equation

$$
\frac{m e^{2}}{\hbar} \frac{1}{(n-1) n}=\frac{m}{\Delta t}(2 n-1) \frac{\hbar^{2}}{m e^{2}}
$$

or

$$
\Delta t=\Delta t_{e m}=\frac{\hbar^{3}}{m e^{4}}(2 n-1)(n-1) n \cong \frac{\hbar^{3}}{m e^{4}} 2 n^{3}
$$

where the last step holds for the large $n$.

On the other hand, for the absorption process in (24), we have the balance

$$
\begin{aligned}
& p^{n+1}-p^{n}+\frac{m}{\Delta t}\left[(n+1)^{2}-n^{2}\right] \frac{\hbar^{2}}{m e^{2}} \\
& =\frac{m e^{2}}{\hbar}\left(\frac{1}{n+1}-\frac{1}{n}\right)+\frac{m}{\Delta t}(2 n+1) \frac{\hbar^{2}}{m e^{2}}=0
\end{aligned}
$$

from which

$$
\frac{m e^{2}}{\hbar} \frac{-1}{(n+1) n}=-\frac{m}{\Delta t}(2 n+1) \frac{\hbar^{2}}{m e^{2}}
$$


or

$$
\Delta t=\Delta t_{a b s}=\frac{\hbar^{3}}{m e^{4}}(2 n+1)(n+1) n \approx \frac{\hbar^{3}}{m e^{4}} 2 n^{3},
$$

where the last step holds for the large $n$.

Evidently

$$
\Delta t_{a b s}-\Delta t_{e m}=\frac{\hbar^{3} n}{m e^{4}}[(2 n+1)(n+1)-(2 n-1)(n-1)]=\frac{\hbar^{3}}{m e^{4}} 6 n^{2} .
$$

For large $n$ the difference (31) becomes only a small fraction of $\Delta t$ in (27) or (30).

\section{Electron Particle Moving in a One-Dimensional Potential Box and Its Transition Process}

A reasoning similar to that developed for the electron in the hydrogen atom can be applied also in case of the electron particle moving in a one-dimensional potential box.

Let the box has the length $L$. The electron quantum states for the energy are $[18]$

$$
E_{n}=\frac{n^{2} h^{2}}{8 m L^{2}}=\frac{m}{2} v_{n}^{2}
$$

or

$$
E_{n}=\frac{p_{n}^{2}}{2 m}
$$

where $v_{n}$ are the electron velocities:

$$
v_{n}=\frac{n h}{2 m L}
$$

and $p_{n}$ are the electron momenta

$$
p_{n}=\frac{n h}{2 L} .
$$

The electron can have the momenta in both motion directions along the box, so $p_{n}$ in (33a) can have both positive and negative values. Let the energy emission produces the difference of momenta equal to that in the states $n$ and $n-1$. This difference becomes:

$$
\Delta p_{n}=p_{n}-p_{n-1}=\frac{h}{2 L}
$$

Due to the momentum conservation the difference (34) should be cancelled by the momentum

$$
m \frac{2 L}{\Delta t}
$$

where $\Delta t$ is the time interval of the electron motion along the box length, first from zero to $L$, next from $L$ to zero. This implies 


$$
\frac{2 L}{\Delta t}=v_{n}
$$

which is the velocity of the particle. This velocity has been obtained from the electron energy in the formula (33).

The momentum balance provides us with the equation

$$
-\Delta p_{n}+m \frac{2 L}{\Delta t}=0
$$

which gives

$$
\Delta p_{n}=\frac{h}{2 L}=m \frac{2 L}{\Delta t}=m v_{n}
$$

where the last step is due to (36).

From (38) we obtain the relation for $\Delta t$ :

$$
\Delta t=\frac{2 L}{v_{n}}=2 L \frac{2 m L}{n h}=\frac{4 m L^{2}}{n h}
$$

identical to that calculated from [19]:

$$
\Delta t=\frac{\Delta q}{\partial E_{n} / \partial p_{n}}=\frac{2 L}{p_{n} / m}=\frac{2 L}{v_{n}}
$$

because the distance $\Delta q=2 L$. This result can be compared with the formula represented by the Joule-Lenz law, viz.

$$
\Delta E \Delta t=h .
$$

Since $\Delta E$ in (40) becomes for a free particle

$$
\Delta E=E_{n}-E_{n-1}=\frac{n^{2}-(n-1)^{2}}{8 m L^{2}} h^{2} \approx \frac{2 n h^{2}}{8 m L^{2}}=\frac{n h^{2}}{4 m L^{2}}
$$

we obtain from (41) the time interval

$$
\Delta t=\frac{h}{\Delta E}=h \frac{4 m L^{2}}{n h^{2}}=\frac{4 m L^{2}}{n h} .
$$

This is a result identical to $\Delta t$ in (39) and (39a).

\section{Size Limits of Mechanical Parameters Entering Simple Quantum Systems}

Conservation of momentum suggests to calculate the limits of mechanical parameters like energy, velocity, distance and time entering the examined simple quantum systems. These limits can be obtained in an equally simple way.

Beginning with the hydrogen atom, the relativistic limit of the electron velocity leads to requirement

$$
\left.v_{n}\right|_{n=1}=v_{1}=\frac{e^{2}}{\hbar}<c
$$

which gives

$$
1<\frac{\hbar c}{e^{2}} \cong 137 .
$$


A limit for the absolute value of the electron energy is represented by the formula

$$
E_{1}=E_{\mathrm{kin}}^{(n=1)}=\frac{m v_{1}^{2}}{2}=\frac{m e^{4}}{2 \hbar^{2}}<m c^{2}
$$

which gives the relation

$$
\frac{e^{4}}{2 \hbar^{2}}<c^{2} .
$$

This relation is equivalent to the formula

$$
1<\frac{2 \hbar^{2} c^{2}}{e^{4}} \cong 2 \cdot 137^{2} .
$$

The result in (47) is an extension of that presented in (44).

The properties connected with the radius limit of the electron orbit which for the quantum number $n=1$ is equal to

$$
r_{n}=r_{1}=\frac{\hbar^{2}}{m e^{2}}
$$

can be deduced from the virial theorem

$$
2 E_{\text {kin }}+E_{\text {pot }}=0 .
$$

This gives the largest negative size of the electron potential energy equal to

$$
E_{\mathrm{pot}}^{(n=1)}=-\frac{e^{2}}{r_{1}}=-2 E_{\mathrm{kin}}^{(n=1)}
$$

coupled with the largest kinetic electron energy value. From (50) we have the relation

$$
\frac{e^{2}}{r_{1}}=2 E_{\text {kin }}^{(n=1)}=m v_{1}^{2} .
$$

Since $v_{1}<c$ we obtain

$$
\frac{e^{2}}{r_{1}}<m c^{2}
$$

or

$$
r_{1}>\frac{e^{2}}{m c^{2}} .
$$

By taking into account (48), we obtain from (52a):

$$
\frac{\hbar^{2}}{m e^{2}}>\frac{e^{2}}{m c^{2}}
$$

according to which

$$
1>\frac{e^{4}}{\hbar^{2} c^{2}} \cong \frac{1}{137^{2}} .
$$

There remains still the condition satisfied by the time interval $\Delta t$ of the electron transition. We have the formula 


$$
\Delta r_{n}=r_{n+1}-r_{n}=\frac{(n+1)^{2}-n^{2}}{m e^{2}} \hbar^{2}=\frac{2 n+1}{m e^{2}} \hbar^{2}
$$

which gives the change of the radius of the electron orbit. For two neighbouring quantum numbers, $n$ and $n+1$, we have

$$
\frac{\Delta r_{n}}{\Delta t}=\Delta v_{n},
$$

where the velocity change satisfies the condition:

$$
\left|\Delta v_{n}\right|=\left|\frac{e^{2}}{(n+1) \hbar}-\frac{e^{2}}{n \hbar}\right|=\frac{n+1-n}{(n+1) n} \frac{e^{2}}{\hbar} \approx \frac{1}{n^{2}} \frac{e^{2}}{\hbar}<\frac{e^{2}}{\hbar}<c
$$

where the last steps hold for any large $n$. An alternative formula for the last step in (57) is:

$$
\frac{\hbar}{e^{2}}>\frac{1}{c} .
$$

We obtain

$$
\Delta t=\frac{\Delta r_{n}}{\left|\Delta v_{n}\right|}=\frac{2 n+1}{m e^{2}} \hbar^{2} \frac{n^{2} \hbar}{e^{2}} \approx \frac{2 n^{3} \hbar^{3}}{m e^{4}} .
$$

For very low $n$, say $n=1$, relation (59) for $\Delta t$ becomes

$$
\Delta t=\frac{3 \hbar^{3}}{m e^{4}}>\frac{3 \hbar}{m c^{2}}
$$

on condition (58) does hold.

A similar reasoning can be performed for the electron particle moving in a one-dimensional potential box. In the first step, from the requirement that the kinetic energy on the quantum level $n=1$ is smaller than the rest energy of the electron particle, we obtain the formula:

$$
E_{n=1}=\frac{h^{2} 1^{2}}{8 m L^{2}}=\frac{m v_{1}^{2}}{2}<m c^{2}
$$

which gives the requirement

$$
v_{1}<2^{1 / 2} c .
$$

Therefore, with the aid of the first equation given in (61), we obtain:

$$
v_{1}=\frac{h}{2 m L}<2^{1 / 2} c .
$$

In effect it should be

$$
\frac{h}{2^{3 / 2} m c}<L .
$$

The limits obtained for $L$ and $v_{1}$ can provide us with the size of the interval $\Delta t$ according to the formula

$$
\Delta t v_{1} \cong 2 L,
$$

so a maximal size of the interval $\Delta t$ for the electron oscillation in the box be- 
comes:

$$
\Delta t \cong \frac{2 L}{v_{1}}=\frac{2 L \cdot 2 m L}{h}=\frac{4 m L^{2}}{h} .
$$

Another approach applies $L$ calculated in (64) and $v_{1}$ in (63):

$$
\Delta t \cong \frac{2 L}{v_{1}} \cong \frac{h}{2^{1 / 2} m c} \cdot \frac{1}{2^{1 / 2} c}=\frac{h}{2 m c^{2}} .
$$

\section{Summary}

In the paper, the transition time between the nearest quantum energy levels is examined for the case of the Bohr hydrogen atom and the electron particle enclosed in a one-dimensional potential box. In both cases, the calculations are based on the assumption that the electron momentum in course of the electron transition is conserved.

It is found that the time intervals of the electron transitions obtained in this way are much similar to those calculated on the basis of the Joule-Lenz law for the energy emission: in the case of hydrogen, a difference between the results of both kinds is represented by a constant factor $\pi$; for the electron particle moving in a one-dimensional potential box there exists an identity of the results for $\Delta t$ calculated in both ways. The limiting sizes of the mechanical parameters characterizing the quantum states in the systems considered in the paper have been also calculated.

It should be noted that the electron transition time $\Delta t$ considered in the paper does not correspond, in general, to the reciprocal time of the frequency $v_{n+q, n}$ joining the energy difference of two quantum states $E_{n+q}$ and $E_{n}$ by the formula

$$
E_{n+q}-E_{n}=h v_{n+q, n}=\frac{h}{T_{n+q, n}} .
$$

Only for the case of

$$
q=1
$$

we have proved the formula

$$
T_{n+1, n}=\Delta t
$$

see [8] [9] [10] [11].

\section{Conflicts of Interest}

The author declares no conflicts of interest regarding the publication of this paper.

\section{References}

[1] Einstein, A. (1917) Physikalische Zeitschrift, 18, 121. https://doi.org/10.1007/BF01809624

[2] Planck, M. (1923) Vorlesungen über Theorie der Wärmestrahlung. Johann Ambro- 
sius Barth, Leipzig.

[3] Planck, M. (1932) Theorie der Wärme. S. Hirzel, Leipzig.

[4] Van der Waerden, B.L. (1967) Sources of Quantum Mechanics. Dover, New York.

[5] Schiff, L.I. (1968) Quantum Mechanics. 3rd Edition, McGraw-Hill, New York.

[6] Slater, J.C. (1960) Quantum Theory of the Atomic Structure. McGraw-Hill, New York.

[7] Lass, H. (1950) Vector and Tensor Analysis. McGraw-Hill, New York. https://doi.org/10.1119/1.1932684

[8] Olszewski, S. (2015) Journal of Modern Physics, 6, 1277-1288. https://doi.org/10.4236/jmp.2015.69133

[9] Olszewski, S. (2016) Journal of Modern Physics, 7, 162-174. https://doi.org/10.4236/jmp.2016.71018

[10] Olszewski, S. (2016) Journal of Modern Physics, 7, 827-851. https://doi.org/10.4236/jmp.2016.78076

[11] Olszewski, S. (2016) Journal of Modern Physics, 7, 1004-1020. https://doi.org/10.4236/jmp.2016.79091

[12] Olszewski, S. (2016) Reviews in Theoretical Science, 4, 336-352. https://doi.org/10.1166/rits.2016.1066

[13] Olszewski, S. (2019) Journal of Modern Physics, 10, 1522-1531. https://doi.org/10.4236/jmp.2019.1013101

[14] Olszewski, S. (2020) Journal of Quantum Information Science, 10, 1-9. https://doi.org/10.4236/jqis.2020.101001

[15] Bohr, N. (1922) The Theory of Spectra and the Atomic Constitution. Cambridge University Press, Cambridge.

[16] Olszewski, S. (2017) Journal of Computational and Theoretical Nanoscience, 14, 3662-3664. https://doi.org/10.1166/jctn.2017.7006

[17] Sommerfeld, A. (1931) Atombau und Spektrallinien. Vol. 1, 5th Edition, Vieweg, Braunschweig.

[18] Eyring, H., Walter, J. and Kimball, G.E. (1957) Quantum Chemistry. Wiley, New York.

[19] Landau, L.D. and Lifshitz, E.M. (1948) The Field Theory. 2nd Edition, OGIZ, Moscow. (In Russian) 